\title{
New facet of honey bees dancing language for mining the induction rules
}

\author{
SUBHASH CHANDRA PANDEY \\ Computer Science and Engineering Department, Birla Institute of Technology, Ranchi, Patna Campus, \\ Patna 800014, India \\ e-mail: subh63@yahoo.co.in
}

MS received 16 February 2017; accepted 27 August 2017; published online 14 March 2018

\begin{abstract}
Artificial Bee Colony (ABC) algorithm is used in many domains of computation, including optimization, clustering and classification tasks. Further, honey bees dancing is one of the most fascinating and intriguing behaviours of animal life. Honey bees" dancing is termed as "waggle Dance" in literature and they perform it for indicating the food sources in their environment. This work presents a novel honey bees dancing language (HBDL)-based algorithm for mining the induction rules from datasets. The proposed HBDL algorithm is implemented and tested against the performance of $\mathrm{ABC}$, Particle Swarm Optimization and nine more traditional algorithms frequently used by researchers. The experimental results showed that HBDL is a suitable and effective technique for data mining and classification task.
\end{abstract}

Keywords. Natural computing; artificial bee colony; honey bees dancing language; data classification.

\section{Introduction}

Natural computing is the field of research that encompasses models and computational techniques inspired by nature. It strives to understand the world around us to utilize natural techniques for the purpose of information processing [1].

There is plethora of biological and natural processes that have substantially influenced the methodologies of science and technology in the past years. During the last decade, nature-inspired intelligence became increasingly popular through the development and utilization of intelligent paradigms in advanced information systems design. Natureinspired approaches have vividly and successfully been applied for different tasks in different walks of science and technology, including optimization within complex domains of data or information. These nature-inspired techniques represent the team behaviour of animal and micro-organisms. There is abundance of work based on Particle Swarm Optimization (PSO) [2], which is inspired by birds flocking or fish schooling. Further, artificial immune systems [3, 4] and ants colony optimization [5, 6], inspired by foraging behaviours of the ants, are the example of team intelligence. In the recent few years a number of swarm intelligence algorithms based on bees behaviour have been presented [7]. These algorithms are divided, mainly, into two categories according to their behaviour in the nature. They are the foraging behaviour and the mating behaviour. The most important approaches that simulate the foraging behaviour of the bees are the Artificial Bee Colony
(ABC) algorithm [8, 9], the Virtual Bee (VB) algorithm [10], the Bee Colony Optimization (BCO) algorithm [11], the Bee Hive (BH) algorithm [12], the Bee Swarm Optimization (BSO) algorithm [13] and the Bees algorithm [14]. The ABC algorithm was incepted in 2005 [15] and since then it has been widely used in many optimization, clustering and classification applications such as protein tertiary structures [16], digital IIR filters [17], artificial neural networks [18] and others. This is because of its naïve concept, easy implementation and fewer control parameters.

The ABC algorithm [8, 9] is, mainly, applied in continuous optimization problems. This algorithm simulates the waggled dance behaviour performed by the bees during the foraging process. Similarly, the VB algorithm [10] is, also, applied in continuous optimization problems and uses the concept of waggle dance in a different manner. Further, in the $\mathrm{BH}$ [12] algorithm, dance language and foraging behaviour of a protocol based on honey bees is used. The concept of waggle dance has also been used in the BSO [13]. In BSO, bees find initial solutions (food sources) and subsequently other solutions are worked out from these initial solutions with certain strategies. The strategy followed is the following: every bee is assigned a solution and when they accomplish their search, the bees communicate among themselves with a waggle dance strategy. Furthermore, the best solution will become the new reference solution. It is worthwhile to mention that in the bees algorithm a population of initial solutions (food sources) is randomly generated. [14]. Now, the bees are assigned to the 
solutions based on their fitness function. The bees return to the hive and based on their food sources, a number of bees are assigned to the same food source in order to find a better neighbourhood solution. In the BCO [11] algorithm, a step by step solution is produced by each employed bee and when the employed bees come back to the hive a waggle dance is performed by each employed bee. Then the onlooker bees, based on a probability, follow the employed bees.

There is one more algorithm, which is based on the marriage behaviour of the bees. In literature it is known as the Honey Bees Mating Optimization (HBMO) algorithm $[19,20]$. This algorithm has also been used in different applications [21-23]. The HBMO algorithm is also used in classification task [24].

Honey bee dancing is perhaps the most intriguing aspect of bees. We can consider it as most fascinating behaviours in animal life. This paper presents a novel and ingenious algorithm using the honey bees dancing language (HBDL) in an entirely different manner. Author use the proposed HBDL algorithm for mining the induction rules from datasets. Rest of the paper is organized as follows. Section 2 provides a brief introduction about the HBDL. Section 3 describes the proposed algorithm and its implementation details. Experimental analysis is given in section 4. Finally, the paper is concluded in section 5 .

\section{Honey bees dancing language}

The honey bees communicate the location of food and other resources through body movements. This body movement is termed as waggle dance. This HBDL is performed by the foragers on their return to the nest. It has been described by many observers over several centuries.

In 1960s, von Frisch published a masterly review of research on the dance language [25]. Since the publication of this review, the honey bees dance language has received in-depth attention of many researchers and consequently they pursued vigorous research on this topic and many new aspects such as understanding of the sensory basis of dance communication has been worked out. Reviews of this more recent research have generally focussed on one or a few aspects of the dance language [26-29]. It has been observed by the researchers that when a successful employed bee returns back to the nest with a rich food source it is greeted by onlooker bees. Further, employed bees regurgitate their load to onlookers. Furthermore, employed bees communicate the location of food source in terms of dance communication [25]. Employed bees dance on the vertical sheet of comb. This dance includes a series of frequent repeated waggling runs. It is observed that in this waggling run the bee moves in a particular direction along the comb while waggling her body from side to side. It has also been noticed that during the waggling run they also emit sound by buzzing their wings. Researchers have observed that after each waggling run, the dancer bees circle around and realign themselves to begin the next waggling run. During the dancing of employed bee, the dancer employed bee is encircled by 1-6 other onlooker bees facing towards the dancer. These onlooker bees keenly follow the dancer's movements. The onlooker bees observe several waggling runs and then leave the nest in search of food. The orientation of waggling run and its duration are highly correlated with direction and distance that employed bee has flown for the food. Figures 1 and 2 show the waggling dance [30].

\subsection{Measurement of distance}

In [25] it is suggested that bees determine their flight distance by measuring the expenditure of energy during the flight. It is also suggested that the weights energy expenditure is more on the outward flight from the nest in comparison with the homeward flight. There are several observations in favour of this conclusion [25, 31]. In [25] an alternative hypothesis of monitoring the optic flow has also been proposed.

\subsection{Measurement of direction}

The sun has been considered as an optimum device for the directional reference since a long time. This is because of its reliability and conspicuousness. However, the sun is also associated with two major difficulties. First, it is sometimes behind the clouds. Second, it moves.

It is believed by many researchers that bees do obtain compass information from the polarization patterns of light coming from the blue sky $[25,32,33]$. These patterns are

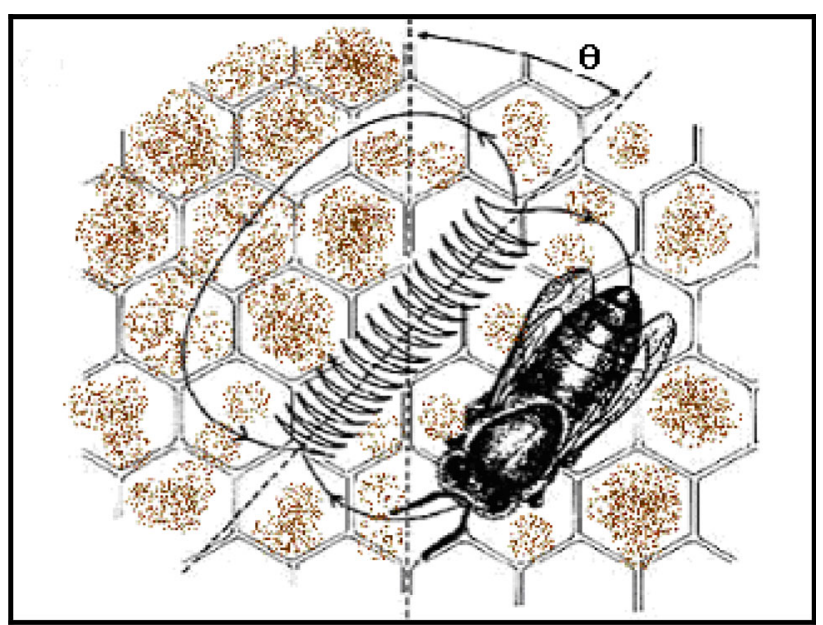

Figure 1. Waggle dance of honey bees. During the flight to food or another resource, honey bees measure the direction (relative to the sun) and distance to the food. 


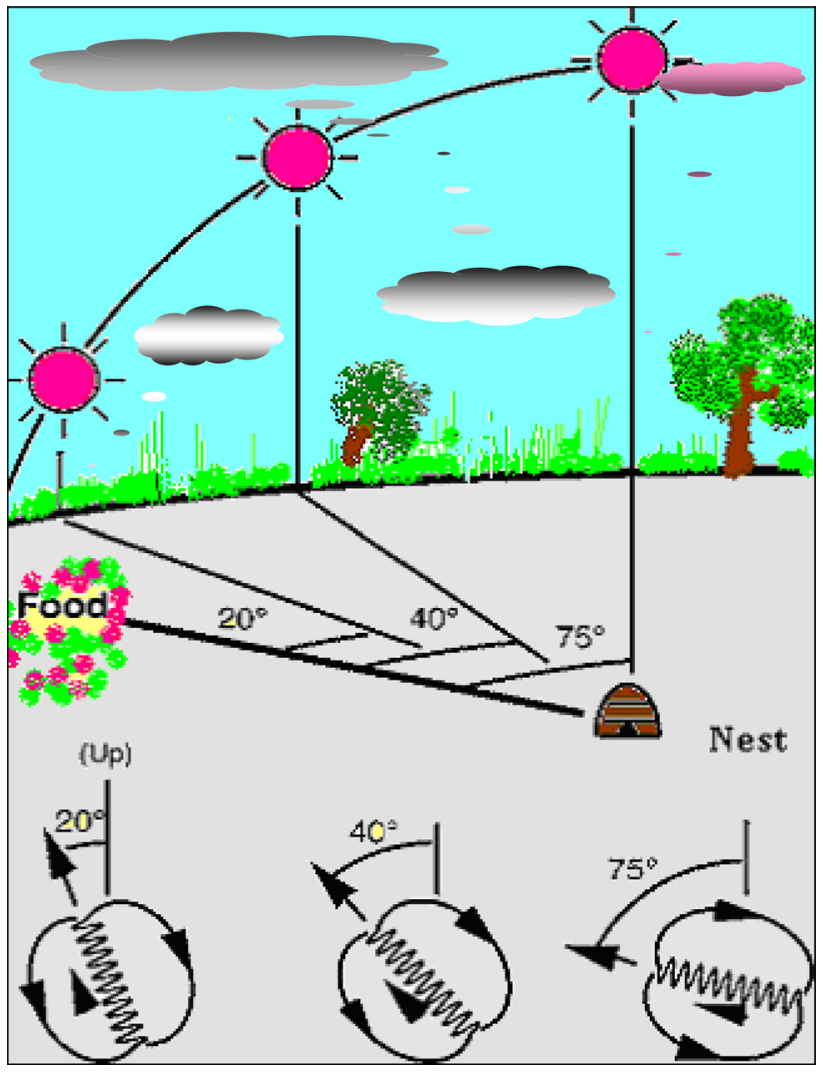

Figure 2. Dance communication as a window on the bee's ability to compensate for changes in the sun's azimuth.

produced when the sun's light is scattered in the atmosphere. Vigorous research works have been conducted by many researchers to find how source of celestial information is used for orientation [32-34]. In [25], Von Frisch studied how bees use the sun for navigation and how a time-compensated sun compass is used by animals. These experiments involved training bees to find food in a particular compass direction and then assessing the accuracy of orientation relative to the sun after various time intervals during which the sun moved [25]. The celestial position of sun at different points of time and its relation with food source are shown in figure 2.

\section{The HBDL algorithm}

Our proposed algorithm borrows its concepts from nature and utilizes the information processing mechanism of dancer bees (employed and scout bees) used for locating the food sources and eventually to communicate it through the waggle dance to the onlooker bees. On the basis of waggle dance performed by the employed and scout bees to convey the spatial information about the food source, author constructed the HBDL algorithm for the purpose of data classification and knowledge discovery. This algorithm is given in figure 3.

\section{The HBDL Algorithm}

1. Load training samples.

2. Generate the initial population of solution (coordinates) of food source based on sun's first celestial position.

3. Set cycle to 1 .

4. repeat

5. FOR each employed bee

\{

Produce new solution (direction and distance) of the food source.

6. Calculate distance using equation (1).

7. Calculate direction cosine using equation (2) from $Z$ axis.

$$
\text { \} }
$$

8. FOR each onlooker bee

\{

Decide the location of food source on the basis of direction and memorize the best direction (best solution).

9. If the information regarding direction and distance provided by employed bees are very similar to the scout bees for different food sources

10. then combine the information of direction and distance to produce the best solution and memorize.

11. If it is still not possible to locate the food then source correctly

\{

$$
\text { Cycle }=\text { Cycle }+1 \text {; }
$$

Evaluate the direction and distance of food source with respect to the next celestial position of the sun and fuse this information with the information previously obtained.

12. Memorize the best solutions so far. \}

13. until all food sources have been located or

$$
\text { Cycle }=L C P
$$

Figure 3. Different steps of the HBDL algorithm.

Similar to the ABC algorithm, the proposed HBDL algorithm also consists of three groups of bees. They are as follows. 1. The employed bees determine the food sources (possible solutions) from a pre-specified set of food sources and share this information through the waggle dance with the other bees in the hive. 2 . The onlooker bees receive the information regarding the food source from the employed bees and they search for a better food source in the 
neighbourhood of the memorized food sources. 3. The scout bees are the employed bees whose food source has been abandoned and now they search for a new food source randomly. The idea of our proposed HBDL algorithm is as follows.

The onlooker bees rely more on the information given by the employed bees. Onlooker bees evaluate the information regarding the direction of the food source provided by the employed bees. If the onlooker bees get confirmation about the food source from the directional information relative to sun azimuth, they memorize it and do fly for its search. Seldom the employed and scout bees provide very similar information for food sources of different regions and this creates confusion before onlooker bees. In such situations, onlooker bees try to locate the food source with the combined information of direction and distance. If this combined information can locate the food source with $100 \%$ conformity they will fly for its search; otherwise, onlooker bees wait and receive more information about these food sources. The onlooker bees observe the information provided by the employed and scout bees pertaining to these confusing food sources at the next celestial position of the sun and fuse this new information with previous one unless the locations of food sources have not been successfully recorded with $100 \%$ surety. The process of including the information is repeated unless the food sources have completely been located or the last celestial position $(L C P)$ of sun has been reached.

\subsection{Basic algorithm structure}

The proposed $H B D L$ framework is introduced in the context of data classification problem. Different steps of the proposed algorithm are schematically presented in figure 3 .

\subsection{Implementation of proposed algorithm}

In this section author will present complete implementation details and assumptions of our proposed algorithm. The proposed algorithm comprises the following assumptions:

\section{2a Assumptions:}

1. Classes are analogous to different regions.

2. Examples or instances are analogous to food sources in those regions.

3. Attributes are similar to the three-dimensional coordinates of these food sources corresponding to different celestial positions of the sun.

4. Distance of food source will be measured from the nest and the nest position will always be fixed at origin, i.e., nest's coordinate will always be $[0,0,0]$.

5. Direction cosine of food source will be measured with respect to $Z$-axis.

6. If numbers of attributes are not an exact multiple of three then we will take extra zeros to make it exact multiple of three.

7. If numbers of attributes are exact multiple of three then we need not put extra zeroes.

8. In this algorithm the sum of employed and scout bees is taken to be equal to the food sources (i.e., total number of examples or instances of the dataset).

9. Sometimes, employed bees and scout bees display almost similar information through waggle dance for food sources of different regions and it creates confusion before onlooker bees.

3.2b Implementation details: We use a sample table 1 to illustrate the idea. This table contains three classes: $\mathrm{X}, \mathrm{Y}$ and $\mathrm{Z}$. There are four attributes: A, B, C and D. The number

Table 1. Sample table.

\begin{tabular}{|c|c|c|c|c|c|c|c|}
\hline \multirow[b]{2}{*}{ Attributes Region } & \multirow[b]{2}{*}{ Food source \# } & \multicolumn{3}{|c|}{$\begin{array}{l}\text { Coordinate of food source at the first celestial } \\
\text { position of sun }\end{array}$} & \multicolumn{3}{|c|}{$\begin{array}{l}\text { Coordinate of food source at the second celestial } \\
\text { position of sun }\end{array}$} \\
\hline & & A & B & $\mathrm{C}$ & $\mathrm{D}$ & & \\
\hline \multirow[t]{5}{*}{ Region X } & 1 & 4.6 & 3.4 & 1.4 & 0.3 & 0 & 0 \\
\hline & 2 & 5.0 & 2.9 & 1.5 & 0.2 & 0 & 0 \\
\hline & 3 & 5.8 & 4.0 & 1.2 & 0.2 & 0 & 0 \\
\hline & 4 & 5.1 & 3.7 & 1.5 & 0.4 & 0 & 0 \\
\hline & 5 & 4.3 & 3.0 & 1.1 & 0.1 & 0 & 0 \\
\hline \multirow[t]{5}{*}{ Region Y } & 1 & 6.5 & 2.8 & 4.6 & 1.5 & 0 & 0 \\
\hline & 2 & 4.9 & 2.4 & 3.3 & 1.9 & 0 & 0 \\
\hline & 3 & 5.1 & 2.7 & 3.9 & 1.8 & 0 & 0 \\
\hline & 4 & 5.0 & 2.0 & 3.5 & 1.0 & 0 & 0 \\
\hline & 5 & 6.3 & 3.3 & 6.0 & 2.5 & 0 & 0 \\
\hline \multirow[t]{5}{*}{ Region Z } & 1 & 5.6 & 2.8 & 4.9 & 2.0 & 0 & 0 \\
\hline & 2 & 6.2 & 2.8 & 4.8 & 1.8 & 0 & 0 \\
\hline & 3 & 5.9 & 3.0 & 5.1 & 2.5 & 0 & 0 \\
\hline & 4 & 5.8 & 2.7 & 1.5 & 1.9 & 0 & 0 \\
\hline & 5 & 6.9 & 3.2 & 5.7 & 2.3 & 0 & 0 \\
\hline
\end{tabular}


of examples or instances in each class is five as given in this sample table.

Step one: In this step we will find the distance of each food source from the nest. Let us consider sample table 1 to make the explanation more explicit. We consider the first instance of the region (Class) $\mathrm{X}$. This is [4.6, 3.4, 1.4, 0.3]. Therefore, as per our assumptions the coordinate of this food source in region $\mathrm{X}$ at the first celestial position of the sun is [4.6, 3.4, 1.4]. Further, at any different time of the day the sun's celestial position will change and thus the coordinates of the food source relative to the sun will also change. This new coordinate of the food source will be obtained from the next triplet of the attribute value. In this sample dataset we have taken only four attributes, so the next triplet cannot be constructed. Therefore, we can consider extra zeros to define the coordinate of the food source at the next celestial position of sun. It will be $[0.3,0,0]$ for the first food source of the region $\mathrm{X}$. The implementation details of step 1 are illustrated in figure 4. Now, we can use Eq. (1) for calculating the Euclidean distance between two points $\left[X_{1}, Y_{1}, Z_{1}\right]$ and $\left[X_{2}, Y_{2}, Z_{2}\right]$ situated in space:

$$
\mathrm{ED}=\sqrt{\left(X_{2}-X_{1}\right)^{2}+\left(Y_{2}-Y_{1}\right)^{2}+\left(Z_{2}-Z_{1}\right)^{2}} .
$$

We have assumed that the nest is situated at the point $\{0,0$, 0]. Therefore, distance of first food source of region $X$ from the nest is $D_{1}=\sqrt{(4.6-0)^{2}+(3.4-0)^{2}+(1.4-0)^{2}}=$ 5.888. Similarly, we can calculate the distances of all other food sources of different regions. The distance of different food sources from the nest is given in table 2 .

Step two: In this step we will find the direction cosine of each food source with respect to $Z$-axis.
Let the end coordinates of a line be $\left[X_{1}, Y_{1}, Z_{1}\right]$ and $\left[X_{2}, Y_{2}, Z_{2}\right]$. Then its direction cosine with respect to $Z$-axis can be calculated using the expression

$$
\frac{Z_{2}-Z_{1}}{D}
$$

$D$ is the distance of food source from the nest. The threedimensional coordinates of first food source in region $\mathrm{X}$ at sun's first celestial position is $[4.6,3.4,1.4]$ and its distance from the nest has already been calculated to be equal to 5.888. Therefore the direction cosine of line joining the nest and the first food source of region $\mathrm{X}$ is $\frac{1.4-0.0}{5.888}=0.2377$. Similarly, we can calculate the direction cosines of different food sources of different regions. These are given in table 2. From table 2, it is obvious that onlooker bees can memorize the following information regarding the food sources on the basis of direction cosines with respect to first celestial position of the sun:

1. If direction cosine $<0.3$ then it is region $X$.

2. If $0.5<=$ direction cosine $<0.6$ then it is region $\mathrm{Y}$.

3. If direction cosine $>=0.6$ then it is region $\mathrm{Z}$.

Further, it should be noted that the information regarding the food sources shown shaded in table 2, i.e., food source 5 of region $\mathrm{Y}$ and food source 2, 4 and 5 of region $\mathrm{Z}$ is confusing. This is because of the very similar information provided by the employed and scout bees through the waggle dance. Therefore, in order to locate these food sources correctly, onlooker bees will incorporate the distance with direction cosine. Sometimes it is also possible that onlooker bees do wait until they cannot get the location of food source confirmed with the direction and distance of food source with respect to the second celestial position of

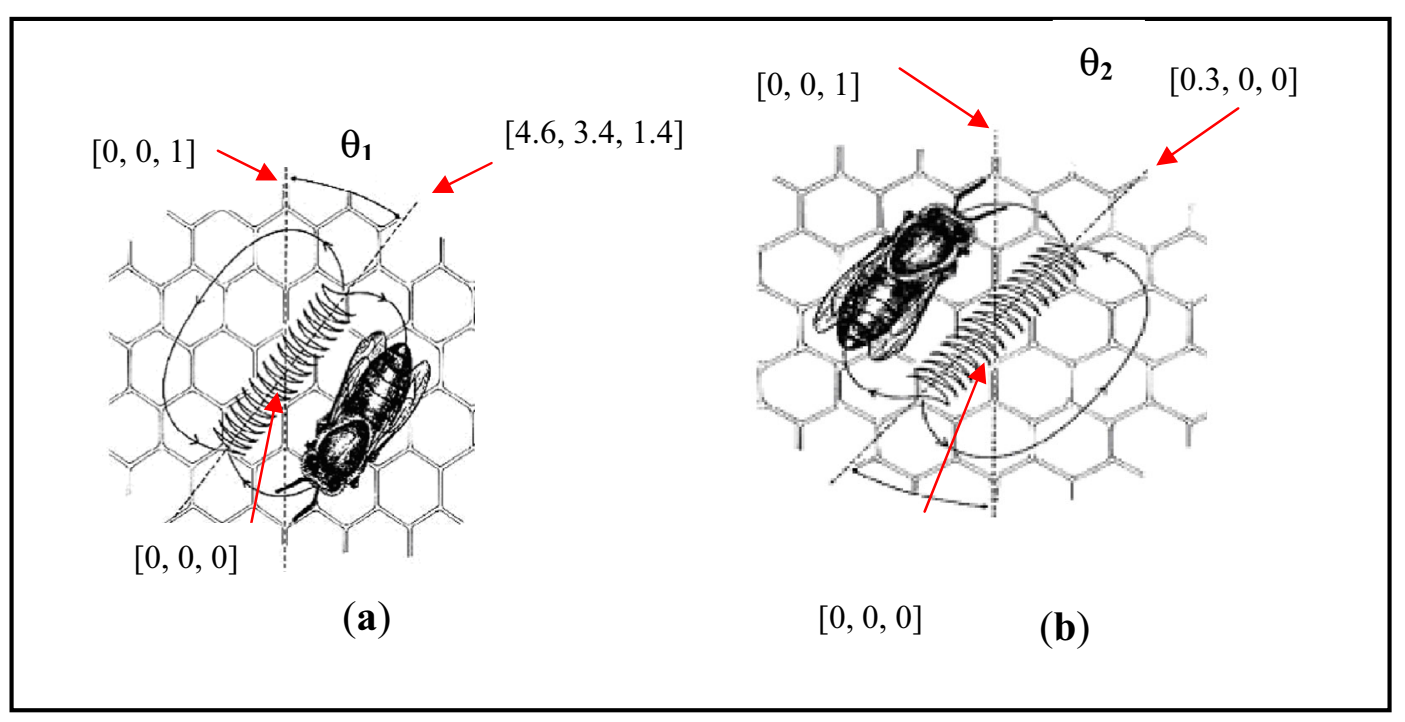

Figure 4. Implementation details of HBDL algorithm: (a) bees position at the first celestial position of sun and (b) bees position at the second celestial position of sun. 
the sun. Therefore, onlooker bees can memorize the following more information for these confusing food sources.

4. If direction cosine and distance of food source at first celestial position of sun are, respectively, 0.6448 and 9.304 then it is region $\mathrm{Y}$.

5. If direction cosine and distance of food source at first celestial position of sun are, respectively, 0.5765 and 8.325 then it is region $\mathrm{Y}$.

6. If direction cosine and distance of food source at first celestial position of sun are, respectively, 0.2282 and 6.571 and distance of the same food source from the nest at second celestial position of the sun is 1.9 then it is region $Z$.

7. If direction cosine and distance of food source at first celestial position of sun are, respectively, 0.5997 and 9.504 and distance of the same food source from the nest at second celestial position of the sun is 2.3 then it is region $Z$.

\subsection{Iris dataset}

In this sub-section we will take the Iris dataset from the UCI machine learning repository [35] to explain the implementation of proposed $H B D L$ framework. This dataset consists of 150 examples of Iris flower and there are three classes: namely, Iris setosa, Iris versicolor and Iris virginica. Each class contains 50 examples. The number of attributes in this dataset is four. They are sepal length, sepal width, petal length and petal width. We can construct the following induction rules for classification of Iris dataset on the basis of direction cosine of food source at first celestial position of sun:
1. If direction cosine of food source $<0.4$ at the first celestial position of the sun then it is setosa.

2. If $0.4<$ direction cosine of food source $<0.6$ at the first celestial position of the sun then it is versicolor.

3. If direction cosine of food source $>0.6$ at the first celestial position of the sun then it is virginica.

Further, the confusing food sources (instances) are given in table 3. This confusion arises before the onlooker bees because employed and scout bees provide very similar information for some food sources, which are actually in different regions. Therefore, onlooker bees will not fly for searching such food sources. Rather, onlooker bees will fuse the information regarding the distance with the direction cosine together to locate the food source more authentically. If it is still not possible to locate the food source with $100 \%$ authenticity the onlooker bees will wait and incorporate the distance and direction cosine information obtained at the next celestial position of the sun with the old information of the same food source. The process of incorporating the information is repeated unless it has not been located with $100 \%$ conformity. Therefore, onlooker bees can construct the following induction rules for the confusing food sources given in table 3 .

4. If direction cosine and distance of food source at the first celestial position of sun are 0.6126 and 8.3246 , respectively, and distance of the same food source from the nest at the second celestial position of sun is 1.6 then it is versicolor.

5. If $0.5<$ direction cosine $<0.6$ and $8.0<$ distance $<10$ for food source at the first celestial position and distance of food source at the second celestial position of sun is 1.8 or 2.0 or 2.1 or 2.3 then it is virginica.

Table 2. Distance and direction of each food source of sample table 1 from the nest.

\begin{tabular}{|c|c|c|c|c|c|}
\hline \multirow[b]{2}{*}{ Region } & \multirow[b]{2}{*}{$\begin{array}{c}\text { Food } \\
\text { source \# }\end{array}$} & \multicolumn{2}{|c|}{ First celestial position of the sun } & \multicolumn{2}{|c|}{ Second celestial position of the sun } \\
\hline & & $\begin{array}{l}\text { Distance of the food } \\
\text { source from nest }\end{array}$ & $\begin{array}{l}\text { Direction cosine of food } \\
\text { source from } Z \text {-axis }\end{array}$ & $\begin{array}{l}\text { Distance of the food } \\
\text { source from nest }\end{array}$ & $\begin{array}{l}\text { Direction cosine of food } \\
\text { source from } Z \text {-axis }\end{array}$ \\
\hline Region & 1 & 5.888 & 0.2377 & 0.3 & 0.0 \\
\hline \multirow[t]{4}{*}{$X$} & 2 & 5.971 & 0.2512 & 0.2 & 0.0 \\
\hline & 3 & 7.147 & 0.1679 & 0.2 & 0.0 \\
\hline & 4 & 6.476 & 0.2315 & 0.4 & 0.0 \\
\hline & 5 & 5.357 & 0.2053 & 0.1 & 0.0 \\
\hline Region & 1 & 8.440 & 0.5450 & 1.5 & 0.0 \\
\hline \multirow[t]{4}{*}{$\mathrm{Y}$} & 2 & 6.376 & 0.5175 & 1.9 & 0.0 \\
\hline & 3 & 6.964 & 0.5600 & 1.8 & 0.0 \\
\hline & 4 & 6.422 & 0.5450 & 1.0 & 0.0 \\
\hline & 5 & 9.304 & 0.6448 & 2.5 & 0.0 \\
\hline Region & 1 & 7.950 & 0.6163 & 2.0 & 0.0 \\
\hline \multirow[t]{4}{*}{$\mathrm{Z}$} & 2 & 8.325 & 0.5765 & 1.8 & 0.0 \\
\hline & 3 & 8.355 & 0.6104 & 2.5 & 0.0 \\
\hline & 4 & 6.571 & 0.2282 & 1.9 & 0.0 \\
\hline & 5 & 9.504 & 0.5997 & 2.3 & 0.0 \\
\hline
\end{tabular}


Table 3. Distance and direction of confusing food sources of Iris dataset from the nest at different celestial positions of the sun.

\begin{tabular}{|c|c|c|c|c|c|}
\hline \multirow[b]{2}{*}{ Class } & \multirow{2}{*}{$\begin{array}{c}\text { Food } \\
\text { source \# } \\
\quad(\# \\
\text { instance) }\end{array}$} & \multicolumn{2}{|c|}{ First celestial position of the sun } & \multicolumn{2}{|c|}{ Second celestial position of the sun } \\
\hline & & $\begin{array}{l}\text { Distance of the food } \\
\text { source from nest }\end{array}$ & $\begin{array}{l}\text { Direction cosine of food } \\
\text { source from } Z \text {-axis }\end{array}$ & $\begin{array}{c}\text { Distance of the food } \\
\text { source from nest }\end{array}$ & $\begin{array}{l}\text { Direction cosine of food } \\
\text { source from } Z \text {-axis }\end{array}$ \\
\hline $\begin{array}{l}\text { Class } \\
\text { versicolor }\end{array}$ & 84 & 8.3246 & 0.6126 & 1.6 & 0 \\
\hline \multirow{9}{*}{$\begin{array}{l}\text { Class } \\
\text { virginica }\end{array}$} & 111 & 8.860 & 0.5756 & 2.0 & 0 \\
\hline & 124 & 8.425 & 0.5815 & 1.8 & 0 \\
\hline & 127 & 8.325 & 0.5765 & 1.8 & 0 \\
\hline & 128 & 8.379 & 0.5847 & 1.8 & 0 \\
\hline & 139 & 8.248 & 0.5819 & 1.8 & 0 \\
\hline & 140 & 9.294 & 0.5810 & 2.1 & 0 \\
\hline & 142 & 9.123 & 0.5590 & 2.3 & 0 \\
\hline & 146 & 8.996 & 0.5780 & 2.3 & 0 \\
\hline & 148 & 8.848 & 0.5876 & 2.0 & 0 \\
\hline
\end{tabular}

\section{Experimental study}

In this work, 13 classification problems are taken from the UCI database [35] to evaluate the performance of HBDL algorithm. Different characteristics of these datasets are given in table 4 . These 13 classification problems are selected as in [36], to make a reliable comparison. Further, we have taken $75 \%$ of data as a training set for the training process and the remaining 25\% of data are used as a test set for the testing purpose. Furthermore, though some datasets' (glass, thyroid and wine) classes are given in sequential list, they are shuffled to represent every class in training as well as in testing. This is similar to the approach taken in [36]. The size of train and test sets can be found in table 4 .

\subsection{Algorithms and settings}

The PSO is an evolutionary algorithm based on population and swarm intelligence. This algorithm simulates the social

Table 4. Characteristics of datasets.

\begin{tabular}{lccrrr}
\hline & Data & Train & Test & Input & Class \\
\hline Balance & 625 & 469 & 156 & 4 & 3 \\
Cancer & 569 & 427 & 142 & 30 & 2 \\
Cancer-int & 699 & 524 & 175 & 9 & 2 \\
Credit & 690 & 518 & 172 & 51 & 2 \\
Dermatology & 366 & 274 & 92 & 34 & 6 \\
Diabetes & 768 & 576 & 192 & 8 & 2 \\
E. coli & 327 & 245 & 82 & 7 & 5 \\
Glass & 214 & 161 & 53 & 9 & 6 \\
Heart & 303 & 227 & 76 & 35 & 2 \\
Horse & 364 & 273 & 91 & 58 & 3 \\
Iris & 150 & 112 & 38 & 4 & 3 \\
Thyroid & 215 & 162 & 53 & 5 & 3 \\
Wine & 178 & 133 & 45 & 13 & 3 \\
\hline
\end{tabular}

behaviour of a flock of birds. We will compare the performance of proposed HBDL algorithm with the results of PSO and ABC as given in [36, 37]. Different parameters of PSO algorithm taken in [36] are $n=50, T_{\max }=1000$, $v_{\max }=0.05, \quad v_{\min }=-0.05, \quad c_{1}=2.0, \quad c_{2}=2.0$, $w_{\max }=0.9$ and $w_{\min }=0.4$. We have also considered the $\mathrm{ABC}$ algorithm for the comparison purpose. Author has taken the results of $\mathrm{ABC}$ algorithm for these datasets as given in [37]. In [37] the values of colony size and maximum cycle number of the $\mathrm{ABC}$ are chosen to be the same as or less than the values of swarm size and maximum iteration number used in PSO [36].

In [36], besides the PSO algorithm, other classification techniques from different groups are considered. These groups are Bayesian, based on functions, lazy, meta-techniques, tree-based and rule-based techniques. For each of those groups, the selected techniques are the BayesNet [38] from the Bayesian; the Multi-Layer Perceptron artificial neural network (MLP) [39] and Radial Basis Function artificial neural network (RBF) [40] from those based on functions; the KStar [41] from the lazy; the Bagging [42]

Table 5. Classification error percentages on test datasets.

\begin{tabular}{lrrr}
\hline & HBDL & ABC [37] & PSO [36] \\
\hline Balance & 13.56 & 15.38 & 25.47 \\
Cancer & 2.67 & 2.81 & 5.80 \\
Cancer-int & 1.34 & 0.00 & 2.87 \\
Credit & 10.07 & 13.37 & 22.96 \\
Dermatology & 4.37 & 5.43 & 5.76 \\
Diabetes & 19.89 & 22.39 & 22.50 \\
E. coli & 13.27 & 13.41 & 14.63 \\
Glass & 40.01 & 41.50 & 39.05 \\
Heart & 12.94 & 14.47 & 17.46 \\
Horse & 37.63 & 38.26 & 40.98 \\
Iris & 0.34 & 0.00 & 2.63 \\
Thyroid & 2.74 & 3.77 & 5.55 \\
Wine & 1.28 & 0.00 & 2.22 \\
\hline
\end{tabular}


Table 6. Average classification error percentages and ranking of the techniques given in [36] and ABC algorithm given in [37] on each problem.

\begin{tabular}{|c|c|c|c|c|c|c|c|c|c|c|c|c|}
\hline & HBDL & $\mathrm{ABC}$ & PSO & BayesNet & MLPann & $\mathrm{RBF}$ & KStar & Bagging & MultiBoost & NBTree & Ridor & VFI \\
\hline \multirow[t]{2}{*}{ Balance } & 13.56 & 15.38 & 25.47 & 19.74 & 9.29 & 33.61 & 10.25 & 14.77 & 24.20 & 19.74 & 20.63 & 38.85 \\
\hline & (3) & (5) & (9) & (6) & (1) & $(10)$ & (2) & (4) & (8) & (6) & (7) & (11) \\
\hline \multirow[t]{2}{*}{ Cancer } & 2.67 & 2.81 & 5.80 & 4.19 & 2.93 & 20.27 & 2.44 & 4.47 & 5.59 & 7.69 & 6.36 & 7.34 \\
\hline & (2) & (3) & (8) & $(5)$ & (4) & $(12)$ & (1) & (6) & (7) & (11) & (9) & (10) \\
\hline \multirow[t]{2}{*}{ Credit } & 10.07 & 13.37 & 22.96 & 12.13 & 13.81 & 43.29 & 19.18 & 10.68 & 12.71 & 16.18 & 12.65 & 16.47 \\
\hline & (1) & (6) & (11) & (3) & (7) & (12) & $(10)$ & (2) & (5) & (8) & (4) & (9) \\
\hline \multirow[t]{2}{*}{ Cancer-int } & 1.34 & 0.00 & 2.87 & 3.42 & 5.25 & 8.17 & 4.57 & 3.93 & 5.14 & 5.71 & 5.48 & 5.71 \\
\hline & (2) & (1) & (3) & (4) & (8) & (12) & (6) & (5) & (7) & (10) & (9) & (10) \\
\hline \multirow[t]{2}{*}{ Dermatology } & 4.37 & 5.43 & 5.76 & 1.08 & 3.26 & 34.66 & 4.66 & 3.47 & 53.26 & 1.08 & 7.92 & 7.60 \\
\hline & (4) & (6) & (7) & (1) & (3) & (10) & (5) & (3) & (11) & (1) & (9) & (8) \\
\hline \multirow[t]{2}{*}{ Diabetes } & 19.89 & 22.39 & 22.50 & 25.52 & 29.16 & 39.16 & 34.05 & 26.87 & 27.08 & 25.52 & 29.31 & 34.37 \\
\hline & (1) & (2) & (3) & (4) & (8) & (12) & (10) & (6) & (7) & (4) & (9) & (11) \\
\hline \multirow[t]{2}{*}{ E. coli } & 13.27 & 13.41 & 14.63 & 17.07 & 13.53 & 24.38 & 18.29 & 15.36 & 31.70 & 20.73 & 17.07 & 17.07 \\
\hline & (1) & (2) & (4) & (6) & (3) & $(11)$ & (9) & (5) & (12) & (10) & $(6)$ & $(6)$ \\
\hline \multirow[t]{2}{*}{ Glass } & 40.01 & 41.50 & 39.05 & 29.62 & 28.51 & 44.44 & 17.58 & 25.36 & 53.70 & 24.07 & 31.66 & 41.11 \\
\hline & (8) & $(10)$ & (7) & (5) & (4) & $(11)$ & (1) & (3) & (12) & (2) & (6) & $(9)$ \\
\hline \multirow[t]{2}{*}{ Heart } & 12.94 & 14.47 & 17.46 & 18.42 & 19.46 & 45.25 & 26.70 & 20.25 & 18.42 & 22.36 & 22.89 & 18.42 \\
\hline & (1) & (2) & (3) & (4) & (7) & (12) & (11) & (8) & (4) & (9) & (10) & (4) \\
\hline \multirow[t]{2}{*}{ Horse } & 37.63 & 38.26 & 40.98 & 30.76 & 32.19 & 38.46 & 35.71 & 30.32 & 38.46 & 31.86 & 31.86 & 41.75 \\
\hline & (7) & (8) & (11) & (2) & (5) & (9) & (6) & (1) & (9) & (3) & (3) & (12) \\
\hline \multirow[t]{2}{*}{ Iris } & 0.34 & 0.00 & 2.63 & 2.63 & 0.00 & 9.99 & 0.52 & 0.26 & 2.63 & 2.63 & 0.52 & 0.00 \\
\hline & (5) & (1) & (8) & (8) & (1) & (12) & (6) & (4) & (8) & (8) & (6) & (1) \\
\hline \multirow[t]{2}{*}{ Thyroid } & 2.74 & 3.77 & 5.55 & 6.66 & 1.85 & 5.55 & 13.32 & 14.62 & 7.40 & 11.11 & 8.51 & 11.11 \\
\hline & (2) & (3) & (4) & (6) & (1) & (4) & (11) & (12) & (7) & (9) & (8) & $(9)$ \\
\hline \multirow[t]{2}{*}{ Wine } & 1.28 & 0.00 & 2.22 & 0.00 & 1.33 & 2.88 & 3.99 & 2.66 & 17.77 & 2.22 & 5.10 & 5.77 \\
\hline & (3) & (1) & (5) & (1) & (4) & (8) & (9) & (7) & (12) & (5) & $(10)$ & (11) \\
\hline
\end{tabular}

and the MultiBoostAB [43] from the meta-techniques; the Naïve Bayes Tree (NBTree) [44] from the tree-based ones; the Ripple Down Rule (Ridor) [45] from the rule-based ones and for the others the VotingFeatureInterval (VFI) [46].

\subsection{Results and discussion}

Author followed the approach given in [37] and for each classification problem the Classification Error Percentage (CEP) has recorded. Indeed, CEP is the percentage of incorrectly classified patterns of the test datasets. The CEP is calculated using Eq. (3) as in [37]:

$$
\mathrm{CEP}=100 \times \frac{\# \text { of misclassified examples }}{\text { size of test dataset }} .
$$

As already mentioned, the dataset is divided in two parts: the training set (the first $75 \%$ ) and the test set (the last $25 \%$ ). The results of our proposed HBDL algorithm, ABC and PSO are given in table 5 in terms of CEP. ABC algorithm outperforms PSO algorithm in 12 classification problems, whereas PSO algorithm's result is better than that of $\mathrm{ABC}$ algorithm only for one problem (the glass problem) in terms of classification error. The proposed HBDL algorithm also outperforms $\mathrm{ABC}$ in 10 problems.
For three problems (the cancer-int, iris and wine) the performance of proposed HBDL is worse than that of the ABC. It is also obvious from this table that the proposed HBDL algorithm outperforms PSO in 12 problems but its performance for glass problem is slightly worse than that of the PSO. Moreover, the average CEPs for all problems are $13.13 \%$ for $\mathrm{ABC}, 15.99 \%$ for PSO and 12.31 for the proposed HBDL.

In table 6, author present the CEP of HBDL algorithm and 11 other techniques. In this table, except for $\mathrm{ABC}$, all the results are taken as given in [36]. The results of $\mathrm{ABC}$ on different classification problems are taken from [37]. This table also represents the rankings of different techniques on each problem inside the parenthesis. It is explicit from table 6 that HBDL algorithm gets the best solution in four of the problems and the second solutions in three of the problems. Further, in order to make a good comparison of the entire algorithms, tables 7 and 8 are also displayed. Table 7 presents the average classification errors of all problems and the general ranking based on the average classification error values. Table 8 presents the sum of the algorithms' rankings of each problem and subsequent ranking based on this total sum.

From table 7 we can infer that the performance of the HBDL technique is best. The MLP artificial neural network 
Table 7. Average classification error percentages and general ranking of the techniques based on average classification on all problems.

\begin{tabular}{lcccccccccccc}
\hline & HBDL & ABC & PSO & BayesNet & MLPann & RBF & KStar & Bagging & MultiBoost & NBTree & Ridor & VFI \\
\hline Average & 12.31 & 13.13 & 15.99 & 13.17 & 12.35 & 26.93 & 14.71 & 13.30 & 22.92 & 14.68 & 15.38 & 18.89 \\
Rank & 1 & 3 & 9 & 4 & 2 & 12 & 7 & 5 & 11 & 6 & 8 & 10 \\
\hline
\end{tabular}

Table 8. The sum of ranking of the techniques and general ranking based on the total ranking.

\begin{tabular}{lcccccccccccc}
\hline & HBDL & ABC & PSO & BayesNet & MLPann & RBF & KStar & Bagging & MultiBoost & NBTree & Ridor & VFI \\
\hline Average & 40 & 50 & 83 & 55 & 56 & 135 & 87 & 66 & 109 & 86 & 96 & 111 \\
Rank & 1 & 2 & 6 & 3 & 4 & 12 & 8 & 5 & 10 & 8 & 9 & 11 \\
\hline
\end{tabular}

is the second best, $\mathrm{ABC}$ is the third best and BayesNet is the fourth best.

Table 8 shows the ranking of different techniques on the basis of the sum of rankings of the techniques on different problems. Table 8 also reflects HBDL as the best technique, $\mathrm{ABC}$ as the second best, BayesNet as the third best and MLP artificial neural network as the fourth best.

\section{Conclusion}

In this work, the HBDL algorithm is proposed. This work can be considered as a new horizon in the ABC domain. The technique is new, simple and robust for mining the induction rules from datasets. The performance of HBDL algorithm is compared to those of the ABC and PSO algorithms. It is also compared to those of nine other techniques widely used by the researchers. Experimental results render the fact that HBDL algorithm can successfully be applied for mining the induction rules from datasets. There are several issues remaining as scopes for future studies such as using different other challenging datasets and techniques and comparing their results with those from the HBDL algorithm.

\section{References}

[1] Rozenberg G 2008 Computer science, informatics and natural computing, personal reflections in new computational paradigms: changing concepts of what is computable. Springer-Verlag, Berlin Heidelberg, pp. 373-379

[2] Kennedy J and Eberhart R 1995 Particle swarm optimization. In: Proceedings of the 1995 IEEE International Conference on Neural Networks, vol. 4, pp. 1942-1948

[3] Dasgupta D (Ed.) 1998 Artificial immune systems and their application. Heidelberg: Springer

[4] De Castro L N and Timmis J 2002 Artificial immune systems: a new computational intelligence approach. Heidelberg: Springer
[5] Dorigo M and Gambardella L M 1997 Ant colony system: a cooperative learning approach to the traveling salesman problem. IEEE Trans. Evol. Comput. 1(1): 53-66

[6] Dorigo M and Stutzle T 2004 Ant colony optimization. Cambridge, Massachusetts-London, England: The MIT Press

[7] Baykasoglu A, Ozbakir L and Tapkan P 2007 Artificial bee colony algorithm and its application to generalized assignment problem. In: Chan F T S and Tiwari M K (Eds.) Swarm intelligence, focus on ant and particle swarm optimization. I-Tech Education and Publishing, Austria, pp. 113-144

[8] Karaboga D and Basturk B 2007 A powerful and efficient algorithm for numerical function optimization: artificial bee colony (ABC) algorithm. J. Global Optim. 39: 459-471

[9] Karaboga D and Basturk B 2008 On the performance of artificial bee colony (ABC) algorithm. Appl. Soft Comput. 8: 687-697

[10] Yang X S 2005 Engineering optimizations via nature-inspired virtual bee algorithms. In: Yang $\mathrm{J} \mathrm{M}$ and Alvarez $\mathrm{J} \mathrm{R}$ (Eds.) IWINAC 2005, LNCS 3562. Berlin-Heidelberg: Springer-Verlag, pp. 317-323

[11] Teodorovic D and Dell'Orco M 2005 Bee colony optimization-a cooperative learning approach to complex transportation problems. In: Advanced OR and AI methods in transportation, pp. 51-60

[12] Wedde H F, Farooq M and Zhang Y 2004 BeeHive: an efficient fault-tolerant routing algorithm inspired by honey bee behaviour. In: Dorigo M (Ed.) Ant colony optimization and swarm intelligence, LNCS 3172, Springer Berlin, pp. 83-94

[13] Drias H, Sadeg S and Yahi S 2005 Cooperative bees swarm for solving the maximum weighted satisfiability problem. In: Proceedings of the IWAAN International Work Conference on Artificial and Natural Neural Networks, LNCS 3512, pp. 318-325.

[14] Pham D T, Ghanbarzadeh A, Koc E, Otri S, Rahim S and Zaidi M 2006 The bees algorithm-a novel tool for complex optimisation problems. In: IPROMS 2006, Proceedings of the 2nd International Virtual Conference on Intelligent Production Machines and Systems. Oxford: Elsevier

[15] Karaboga D 2005 An idea based on honey bee swarm for numerical optimization. TECHNICALREPORT-TR06

[16] Bahamish H A A, Abdullah R and Salam R A 2009 Protein tertiary structure prediction using artificial bee colony 
algorithm. Paper Presented at Modelling and Simulation, AMS '09, Third Asia International Conference on Modelling and Simulation

[17] Karaboga N A 2009 New design method based on artificial bee colony algorithm for digital IIR filters. J. Franklin Inst. 346(4): $328-348$

[18] Karaboga D and Akay B B 2005 An artificial bee colony (abc) algorithm on training artificial neural networks. Technical Report TR06, Erciyes University, Engineering Faculty, Computer Engineering Department

[19] Abbass H A 2001 A monogenous MBO approach to satisfiability. In: Proceedings of the International Conference on Computational Intelligence for Modelling, Control and Automation, CIMCA'2001, Las Vegas, NV, USA

[20] Abbass H A 2001 Marriage in honey-bee optimization (MBO): a haplometrosis polygynous swarming approach. In: Proceedings of the Congress on Evolutionary Computation (CEC2001), Seoul, Korea, pp. 207-214

[21] Afshar A, Haddad O B, Marino M A and Adams B J 2007 Honey-bee mating optimization (HBMO) algorithm for optimal reservoir operation. J. Franklin Inst. 344: 452-462

[22] Fathian M, Amiri B and Maroosi A 2007 Application of honey bee mating optimization algorithm on clustering. Appl. Math. Comput. 190: 1502-1513

[23] Haddad O B, Afshar A and Marino M A 2006 Honey-bees mating optimization (HBMO) algorithm: a new heuristic approach for water resources optimization. Water Resour. Manage. 20: 661-680

[24] Marinaki M, Marinakis Y and Zopounidis C 2010 Honey Bees Mating Optimization algorithm for financial classification problems. Appl. Soft Comput. 10: 806-812

[25] Von Frisch K 1967 The dance language and orientation of bees. Cambridge, MA: Harvard University Press, p. 566

[26] Dyer F C 1991 Comparative studies of dance communication: analysis of phylogeny and function. In: Smith D R (Ed.) Diversity in the Genus Apis, pp. 177-198. Boulder, CO: Westview, $265 \mathrm{pp}$

[27] Gould J L 1976 The dance-language controversy. Q. Rev. Biol. 51: 211-244

[28] Gould J L and Gould C G 1988 The honey bee. New York: Scientific American Library, p. 239

[29] Gould J L, Dyer F C and Towne W F 1985 Recent progress in the study of the dance language. Fortschr. Zool. 31 141-161

[30] Dyer F C 2002 The biology of the dance language. Annu. Rev. Entomol. 47 917-949

[31] Esch H E and Burns J E 1996 Distance estimation by foraging honeybees. J. Exp. Biol. 199: 155-162
[32] Wehner R 1982 Himmelsnavigation bei Insekten, Neurophysiologie und Verhalten. Neujahrsbl. Naturforsch. Ges. Z̈urich 184: 1-132

[33] Wehner R and Rossel S 1985 The bee's celestial compass-a case study in behavioral neurobiology. Fortschr. Zool. 31: $11-53$

[34] Brines M L 1978 Skylight polarization patterns as cues for honey bee orientation; physical measurements and behavioral experiments. $\mathrm{PhD}$ Thesis, The Rockefeller University, p. 389

[35] Blake C L and Merz C J 1998 University of California at Irvine Repository of Machine Learning Databases. http:// www.ics.uci.edu/mlearn/MLRepository.html

[36] De Falco I, Della Cella A and Tarantino E 2007 Facing classification problems with Particle Swarm Optimization. Appl. Soft Comput. 7(3): 652-658

[37] Karaboga D and Ozturk C 2011 A novel clustering approach: Artificial Bee Colony (ABC) algorithm. Appl. Soft Comput. 11: $652-657$

[38] Jensen F 1996 An introduction to Bayesian networks. UCL Press-Springer-Verlag, UK

[39] Rumelhart D E, Hinton G E and Williams R J 1986 Learning representation by back propagation errors. Nature 323: 533-536

[40] Hassoun M H 1995 Fundamental of artificial neural networks. Cambridge, MA: The MIT Press

[41] Cleary J G and Trigg L E $1995 \mathrm{~K}^{*}$ : an instance-based learner using an entropic distance measure. In: Proceedings of the $12^{\text {th }}$ International Conference on Machine Learning, pp. 108-114

[42] Breiman L 1996 Bagging predictors. Mach. Learn. 24(2): 123-140

[43] Webb G I 2000 Multiboosting: a technique for combing boosting and wagging. Mach. Learn. 40(2): 159-196

[44] Kohavi R 1996 Scaling up the accuracy of naïve-Bayes classifiers: a decision tree hybrid. In: Simoudis E, Han J W and Fayyad U (Eds.) Proceedings of the Second International Conference on Knowledge Discovery and Data Mining. AAAI Press, USA, pp. 202-207

[45] Compton P and Jansen R 1988 Knowledge in context: a strategy for expert system maintenance. In: Barter C J and Brooks M J (Eds.) Proceedings of Artificial Intelligence, Adelaide, Australia, vol. 406, LNAI. Berlin: Springer-Verlag, pp. 292-306

[46] Demiroz G and Guvenir A 1997 Classification by voting feature intervals. In: Proceedings of the Seventh European Conference on Machine Learning, pp. 85-92 